\title{
Is There Possibility Of Withdrawal Seizure In Opioid Dependence Syndrome? A Case Report
}

\author{
Regmi B ${ }^{1}$, Limbu $\mathbf{S}^{2}$, Nepal $\mathbf{S}^{2}$ \\ 1. Resident, Department of Psychiatry, B. P. Koirala Institute of Health Sciences, Dharan 2. Assistant \\ Professor, Department of Psychiatry, B. P. Koirala Institute of Health Sciences, Dharan \\ E-mail *Corresponding author : binita2399regmi@gmail.com
}

\section{Abstract}

Opioids are commonly used and abused substance worldwide. Opioid withdrawal may manifest as severe muscle cramps, diarrhea, rhinorrhea, lacrimation, piloerection, yawning, and fever. Here, we report a 32 year old man with heroin dependence, presented with new onset generalized tonic-clonic seizure following heroin withdrawal. Seizure is a life-threating condition and rare phenomenon in opioid withdrawal therefore, reporting of this case is important. It may help clinician to be aware and consider seizure as a part of opioid withdrawal.

Keywords: Locus ceruleus, Opioid dependence, Opioid withdrawal, Seizure

\section{INTRODUCTION}

Active ingredient of opium is the alkaloid morphine. ${ }^{1}$ Heroin (diaacetylmorphine) is the most commonly abused opioid. ${ }^{2}$ Cessation or reduction in opioid use that has been heavy and prolonged use or administration of opioid antagonist after a period of opioid use results in opioid withdrawal. ${ }^{3}$ Opioid withdrawal consists of severe muscle cramps and bone aches, profuse diarrhea, abdominal cramps, rhinorrhea, lacrimation, piloerection, yawning, fever, pupillary dilation, hypertension, tachycardia, and temperature dysregulation, including hypothermia and hyperthermia. ${ }^{2}$ Seizures are less commonly observed in opioid withdrawal. The link between seizures and opioid withdrawal is not well studied in human. There are few case reports available on complicated opioid withdrawal.4,5,6 Here we present a case of new onset seizure in opioid withdrawal.

\section{CASE - HISTORY}

32 years old married male patient belonging to middle socioeconomic status presented in OPD via emergency with 3 episodes of generalized tonic clonic seizures within 2-4 hour intervals. The patient had been a regular opioid user for past 16 years. Along with opioid, he also had history of cannabis use for few years but did not continue it for long. He initially used to take opioid in intravenous form which contained a combination of - diazepam, buprenorphine and promethazine. Tolerance, withdrawal and craving could be established. Two years back, after infection on injection sites, patient stopped use of intravenous form but started taking brown sugar (street form of heroin) by inhalation which further increased in past 2 years. His daily dose was 3-4 gm/day, from last 6 months, had been consuming 6-7 gm daily. He had consumed $7 \mathrm{gm}$ of brown sugar on night time, due to unavailability, was not able to consume next day morning on his usual time. After 12-14 hours of last intake, he had 3 episodes of generalized tonic clonic seizure, associated with tongue bite, frothing, rigid posture and post ictal drowsiness. Each episode lasted for 2-3 minutes and each seizure episodes occurred after 2 hours interval. There was no report of fever, head trauma, recent alcohol or other drug use or any psychiatric or neurological illness. No family history of seizure disorder or psychiatric illness was reported.

On examination, rhinorrhea, moist eyes, yawning, piloerection, mid- dilated pupil, tremor, tachycardia and agitation was noted. Systemic examination was normal, plantar was down going, Kernig's, Brudzinski and nuchal rigidity test was negative. Detailed neurological examination could not be performed as he was refusing due to muscle cramps and body ache. He was admitted to Substance dependence 
treatment ward for detoxification and management purpose.

Baseline investigations to rule out other etiology (blood glucose, calcium, electrolytes, complete blood count, liver function tests, and kidney function tests) along with non-contrast computed tomography head scan and electroencephalography were done and reports were within normal limits.

During inpatient care, no seizure episode was observed. Patient was treated with Tramadol and Codeine phosphate. Daily monitoring was done on clinical opioid withdrawal scale and drugs was gradually tapered and stopped. Motivation enhancement and relapse prevention strategies were discussed. Finally based on the above findings, patient was discharged with diagnosis of opioid dependence syndrome presented in withdrawal with withdrawal seizure.

Patient could not come for follow up due to lockdown in the country, but on contacting through telephone, patient has no further seizure episode. He relapsed into opioid use after remaining abstinent for 2 months.

\section{DISCUSSION:}

Opioid abuse is a serious clinical and social problem. The increasing high incidence of opioids use is because of its strongly inducing adaptations, features of addiction, including tolerance, withdrawal and processes that contribute to compulsive use and relapse. ${ }^{7}$ Morphine and heroin withdrawal syndrome begins 6 to 8 hours after the last dose, usually after a 1- to 2-week period of continuous use or after the administration of a narcotic antagonist. The withdrawal syndrome reaches its peak intensity during the second or third day and subsides during the next 7 to 10 days, but some symptoms may persist for 6 months or longer. ${ }^{2}$ Neurobiologically, opioid withdrawal symptoms occur from changes in activity over locus ceruleus. Locus ceruleus produces Nor Adrenaline (NA). When heroin is discontinued after long use, drugs' inhibitory effect is lost. Increased production of Adenosine Triphosphate (ATP), high level of cyclic adenosine monophosphate and excess NA release occur. As a result adrenergic over activation occur due to unopposed firing of locus ceruleus. Thus, individual experiences jitters, seizure, anxiety and muscle cramps and other withdrawal symptoms.6,7,8 Furthermore, structural changes in neurons can occur due prolonged exposure to heroin which may also contribute to withdrawal symptoms such as seizures. ${ }^{9,10}$

Seizure can occur commonly due to various underlying etiologies like metabolic causes, uremia, infectious disease, brain tumor, trauma, withdrawal from drugs etc. It can also occur as a part of epilepsy syndrome. In our patient, through investigations, neuroimaging and EEG, we could not find other secondary causes for seizure, so our etiology for seizure was directed towards opioid withdrawal. Other idiopathic epilepsy syndrome could not be established as there was no history of childhood or adolescent onset seizure, family history or other features that would suggest epilepsy syndrome.

There are few literatures reporting seizure following opioid withdrawal. Infants born to opioid dependent mothers are found to have epileptiform activity with seizures that may be generalized and myoclonic. ${ }^{11}$ As per our knowledge, no such case with seizure has been reported in our country, though opioid use is common among substance user in Nepal. Aich et al study among opioid users in India and Nepal reported of 2 patients with opioid withdrawal delirium in Nepal. ${ }^{12}$ However, one of the study in India reported seven patients with opioid dependence admitted in the deaddiction center developed convulsions and delirium during the withdrawal phase. ${ }^{13}$ Similarly, there is another case report in Srilanka, where patient presented with new onset seizure during acute opioid withdrawal. ${ }^{6}$

Clinician should be cautious while managing opioid withdrawal, as occurrence of seizure might lead to morbidity and even mortality. All causative factors should be ruled out for seizure, baseline investigations along with neuroimaging and electroencephalography is must for any possible cause of seizure. Management require continuous monitoring, pharmacological therapy including long acting opioid agents for short term. Prophylactic anticonvulsant is not indicated if seizure is due to opioid withdrawal.6,14 Further studies or researches can be done to determine the prevalence of seizure as opioid withdrawal features. 


\section{CONCLUSION:}

Seizure in opioid withdrawal, though uncommon, can occur in opioid dependence. So clinician treating such patient should be vigilant, and perform necessary investigations and manage appropriately.

\section{REFERENCES:}

1. Lowinson J, Ruiz P, Millman R, Langrod J. Opiates: clinical aspects. In: Substance Abuse A Comprehensive Textbook. 4th edition. Lippincott Williams $\mathcal{E}$ Wilkins. 2005. $165 p$.

2. Sadock BJ, Sadock VA, Wolter K. Substance use and addictive disorders. Opioids related disorders. In: Kaplan E Sadock's Synopsis of Psychiatry. 11th Edition. 2015. 659 p.

3. American Psychiatric Association. DSM-5 Diagnostic Classification. In: Diagnostic and Statistical Manual of Mental Disorders. American Psychiatric Association; 2013.

4. Sourav K, Tathagata M, Christoday K. RJ. Does withdrawal seizure occur in opioid dependence syndrome? A case series. Vol. 69, Psychiatry and Clinical Neurosciences. 2015. p. 238.

5. Jain S, Singhai K, Swami M. Seizure as a primary presentation in opioid withdrawal. Vol. 72, Psychiatry and Clinical Neurosciences. 2018. p. 802-3.

6. Basnayake DB, Kannangara T, Wickramasinghe $R$, Wijesena N. A patient with heroin withdrawal presenting with new onset seizures; a case report. Vol. 11, Anuradhapura Medical Journal. 2018. p. 16.

7. Christie MJ. Cellular neuroadaptations to chronic opioids: Tolerance, withdrawal and addiction. $\mathrm{Br} J$ Pharmacol. 2008;154(2):384-96.

8. Kosten TR, Baxter LE. Review article: Effective management of opioid withdrawal symptoms: A gateway to opioid dependence treatment. Am J Addict. 2019;28(2):55-62.

9. Rasmussen K, Beitner-Johnson DB, Krystal JH, Aghajanian GK, Nestler EJ. Opiate withdrawal and the rat locus coeruleus: behavioral,electrophysiological, and biochemical correlates. J Neurosci. 1990;10(7):2308-17.

10. Sklair-Tavron L, Shi WX, Lane SB, Harris HW, Bunney BS, Nestler EJ. Chronic morphine induces visible changes in the morphology of mesolimbic dopamine neurons. Proc Natl Acad Sci USA. 1996;93(20):11202-7.

11. Herzlinger RA, Kandall SR, Vaughan HG. Neonatal seizures associated with narcotic withdrawal. J Pediatr. 1977;91(4):638-41.

12. Aich T, Saha I, Ram D, Ranjan S, Subedi S. A Comparative Study On 136 Opioid Abusers In India and Nepal. J Psychiatr Assoc Nepal. 2014;2(2):11-7.

13. Parkar S, Seethalakshmi R, Adarkar S, Kharawala S. Is this complicated opioid withdrawal? Vol. 48, Indian journal of psychiatry. 2006. p. 121-2.

14. John BCM. Seizure and substance abuse: Treatment considerations. Am Acad Neurol. 2006;67(12 suppl 4):s45-8. 